Research Article

\title{
Theoretical Study of Excitonic Complexes in GaAs/AlGaAs Quantum Dots Grown by Filling of Nanoholes
}

\author{
Mohamed Omri, ${ }^{1}$ Amor Sayari $\mathbb{I}^{2,3}$ and Larbi Sfaxi ${ }^{4,5}$ \\ ${ }^{1}$ DeanShip of Scientific Research (DSR), King Abdulaziz University, Jeddah 21589, Saudi Arabia \\ ${ }^{2}$ Department of Physics, Faculty of Science, University of Jeddah, P.O. Box 80327, Jeddah 21589, Saudi Arabia \\ ${ }^{3}$ Université de Tunis El Manar, Faculté des Sciences de Tunis, Unité de Recherche Spectroscopie Raman UR13ES31, \\ Tunis 2092, Tunisia \\ ${ }^{4}$ High School of Sciences and Technology of Hammam Sousse, Université de Sousse, Sousse, Tunisia \\ ${ }^{5}$ Laboratoire de Micro-Optoélectroniques et Nanostructures de la Faculté des Sciences de Monastir, Université de Monastir, \\ Monastir, Tunisia
}

Correspondence should be addressed to Amor Sayari; amor.sayari@laposte.net

Received 19 June 2021; Accepted 11 August 2021; Published 30 August 2021

Academic Editor: Da-Ren Hang

Copyright (c) 2021 Mohamed Omri et al. This is an open access article distributed under the Creative Commons Attribution License, which permits unrestricted use, distribution, and reproduction in any medium, provided the original work is properly cited.

\begin{abstract}
In this work, a theoretical study of the electronic and the optical properties of a new family of strain-free GaAs/AlGaAs quantum dots (QDs) obtained by AlGaAs nanohole filling is presented. The considered model consists of solving the three-dimensional effective-mass Schrödinger equation, thus providing a complete description of the neutral and charged complex excitons' fine structure. The QD size effect on carrier confinement energies, wave functions, and $s$-p splitting is studied. The direct Coulomb interaction impact on the calculated $s$ and $p$ states' transition energies is investigated. The behaviour of the binding energy of neutral and charged excitons $\left(X^{-}\right.$and $\left.X^{+}\right)$and biexciton $X X$ versus QD height is studied. The addition of the correlation effect allows to explain the nature of biexcitons often observed experimentally.
\end{abstract}

\section{Introduction}

Optimizing the optical qualities of quantum dots (QDs) remains a challenge for researchers. For this purpose, new GaAs/AlGaAs semiconductor QDs have been grown using a new technique based on filling self-assembled nanoholes obtained by local etching of droplets $[1,2]$. This method provides QDs with particular structural properties such as unstrained, uniform, not very dense, with high symmetry, and of different shapes with reference to conventional strained QDs. Due to these characteristics, the optical properties of unstrained QDs are interesting for various increasing high-performance applications in optoelectronic devices, namely, lasers and solar cells [3, 4], in addition to quantum cryptography [5].

In this context, we provide a modelling of the GaAs/ AlGaAs QDs' shape reported by Heyn et al. [2], compatible with available atomic force microscopy (AFM) images. The theoretical study in this paper will focus on the dependence of the confinement energy of the carriers, for the ground state $s$ and state $p$, on the QD height $\left(h_{\mathrm{QD}}\right)$. The calculations are performed by adapting a simple configuration based on BenDaniel and Duke, Hamiltonian [6], which requires much computation time. The obtained results will be compared to the literature experimental results for validation. In addition, our theoretical approach will be compared with Graf et al.'s 
[1] model. Thereby, we will then estimate the effect of the QDs' size on the binding energy of the excitonic complexes in terms of direct Coulomb interaction and correlation effects. Therefore, the final alignment of excitonic states will be explained.

\section{Theoretical Model}

The electronic states of a QD strongly depend on the shape chosen for the dot and its symmetry. In the literature, unstrained self-assembled QDs have been modelled by different shapes such as cone $[7,8]$, pyramid with a square base $[9,10]$, and lens $[11,12]$. The electronic structure of QDs has been calculated by adopting various approaches such as the pseudo-potential model proposed by Williamson and Zunger [13, 14], the strong bond model suggested by Lee et al. [15], and the formalism of the envelope function at several bands by Stier et al. [16].

In this paper, calculations are limited to the formalism of the envelope function with one band. This method consists in choosing a large quantization box, containing the studied physical system (QD), and having all the possible symmetries of the system (Figure 1). This quantization box is a cylinder of radius $R$ and height $H$. These dimensions are chosen such as $R=4 r_{\text {nanohole }}$ and $H=4 h_{\text {nanohole }}$ to avoid side effects. The parameters $r$ and $h$ are, respectively, the radius and height of the QD (Figure 1). The Hamiltonian of each electron $(e)$ or hole $(h)$ is written in the form

$$
\mathrm{H}_{e(h)}=\nabla\left(-\frac{\hbar^{2}}{2 m_{e(h)}^{*}\left(\mathbf{r}_{e(h)}\right)}\right) \nabla+V_{e(h)}\left(\mathbf{r}_{e(h)}\right),
$$

where $m_{e(h)}^{*}$ and $V_{e(h)}$ are, respectively, the effective mass of the electron or hole and the potential for conduction and valence offset. $\mathbf{r}_{e}$ and $\mathbf{r}_{h}$ are the position vectors of electrons and holes inside the cylinder. The offset potential depends on the geometry of the QD; thus, we consider that the confinement potential has a Gaussian shape, and we describe it as follows:

$$
V_{e, h}(r, z)= \begin{cases}0 & \text { if } r \leq r_{\mathrm{QD}} \text { and } z_{\text {inf }}(r)<z(r)<z_{\text {sup }}(r), \\ V_{e, h}, & \text { elsewhere, }\end{cases}
$$

with $\quad z_{\text {inf,sup }}(r)=\sqrt{H_{\text {inf,sup }}^{2}\left[1-\left(r / r_{\mathrm{QD}}\right)^{2}\right]}$ $h_{\mathrm{QD}}=H_{\text {sup }}-H_{\text {inf }}$.

To access the energy levels of QDs, we used the matrix method initially proposed by Marzin and Bastard [8]. Due to the cylindrical symmetry of the QDs, the wave function is expressed as a Fourier-Bessel series written as

$$
\psi=\sum_{n m l} A_{n m l} \Phi_{n m l}
$$

where $A_{n m l}$ are the coefficients to be determined and $\Phi_{n m l}$ are periodic and orthogonal functions which are given by

$$
\begin{aligned}
\Phi_{n m l} & =R_{n m}(r) Z_{l}(z) \theta_{m}(\phi), \\
R_{n m}(r) & =\frac{\sqrt{2}}{R J_{m+1}\left(k_{n m} R\right)} J_{m}\left(k_{n m} r\right), \quad \text { for } 0 \leq r \leq R, \\
Z_{l}(z) & =\sqrt{\frac{2}{H}} \sin \left(l \pi\left(\frac{1}{2}-\frac{z}{H}\right)\right), \quad \text { for }-\frac{H}{2} \leq z \leq \frac{H}{2}, \\
\theta_{m}(\phi) & =\frac{1}{\sqrt{2 \pi}} e^{i m \phi}, \quad \text { for } 0 \leq \phi \leq 2 \pi,
\end{aligned}
$$

where $n, m$, and $l$ are integers and $J_{m}$ is the Bessel function of order $m$ and $k_{n m} r$ is its $n^{\text {th }}$ root.

The matrix element is defined as

$$
H_{n m l, n^{\prime} m^{\prime} l^{\prime}}=\int_{\Omega} \Phi_{n m l}^{*}\left(\nabla\left(-\frac{\hbar^{2}}{2 m^{*}}\right) \nabla+V\right) \Phi_{n^{\prime} m^{\prime} l^{\prime}} \mathrm{d} \Omega,
$$

with $\Omega$ being the cylinder volume. The energy levels and wave functions are calculated using the parameters listed in Table 1.

The AlGaAs nanoholes are modelled by holes of the same shape and dimensions as those of [1]. Nanoholes of radius $r_{\text {nanohole }}=30 \mathrm{~nm}$ and depth $h_{\text {nanohole }}=16 \mathrm{~nm}$ have been considered in our calculations.

The excitonic energies of ground state $s$ and excited state $p$ are, respectively, denoted by $E_{X}^{s}$ and $E_{X}^{p}$. Their expressions are given by

$$
E_{X}^{s(p)}=E_{g}+E_{1(2)}+H H_{1(2)}+J_{e h}^{s(p)},
$$

where $E_{g}$ is the GaAs QD band gap energy. $E_{i}$ and $H H_{i}$ are the confinement energies of the electron and hole $(i=1$ for ground state $s$ and $i=2,3,4, \ldots$, for exited states $p, d, f, \ldots$, respectively). $J_{e h}^{s(p)}$ is the electron-hole Coulomb energy, in the $s$ or $p$ state, treated in the perturbative approach, and it is written as

$$
J_{e h}^{s(p)}=-\frac{e^{2}}{4 \pi \varepsilon_{o} \varepsilon_{r}} \int_{\Omega_{e}} \int_{\Omega_{h}}\left|\Psi_{e}^{s(p)}\right|^{2} \frac{1}{\left|\mathbf{r}_{e}-\mathbf{r}_{h}\right|}\left|\Psi_{h}^{s(p) 2}\right| \mathrm{d} \Omega_{e} \mathrm{~d} \Omega_{h},
$$

with $\varepsilon_{r}$ being the relative dielectric constant of GaAs [17] and $\Omega_{e}$ and $\Omega_{h}$ representing the volume of the cylinder associated with electrons and holes, respectively.

The recombination energies of the neutral exciton $\left(E_{X}\right)$, charged excitons $\left(E_{X^{+}}, E_{X^{-}}\right)$, and biexciton $\left(E_{X X}\right)$ are calculated as follows [18]:

$$
\begin{aligned}
E_{X} & =E_{g}+E_{1}+H H_{1}-\left|J_{e h}^{s}\right|, \\
E_{X^{+}} & =E_{g}+E_{1}+H H_{1}-2\left|J_{e h}^{s}\right|+J_{h h}^{s}, \\
E_{X^{-}} & =E_{g}+E_{1}+H H_{1}-2\left|J_{e h}^{s}\right|+J_{e e}^{s}, \\
E_{X X} & =E_{g}+E_{1}+H H_{1}-3\left|J_{e h}^{s}\right|+J_{e e}^{s}+J_{h h}^{s} .
\end{aligned}
$$

The binding energies of the trions $\Delta_{X^{+}}, \Delta_{X^{-}}$and the biexciton $\Delta_{X X}$, without correlation effects, are estimated within the framework of the Hartree-Fock approximation and defined by 


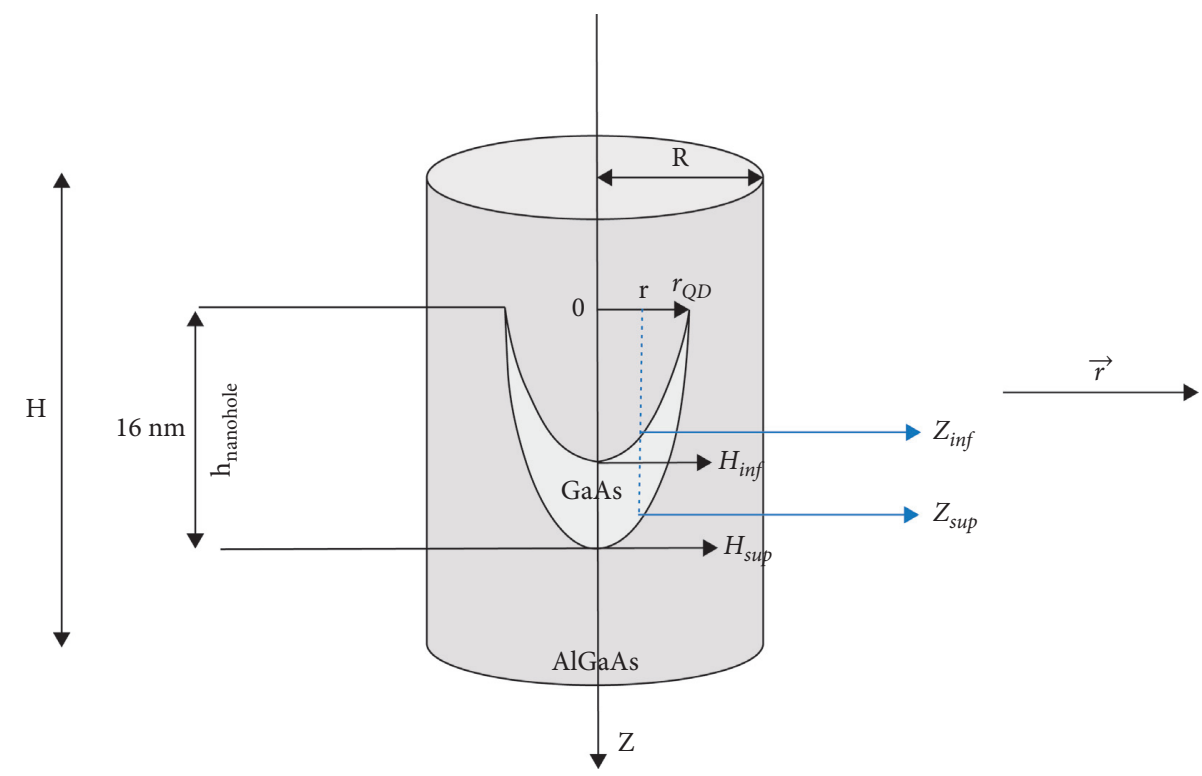

FIGURE 1: Schematic illustration of the shape modelling of the GaAs QD obtained by filling the AlGaAs nanohole.

TABLE 1: Numerical values of the input parameters of the $\mathrm{Al}_{0.36} \mathrm{Ga}_{0.44} \mathrm{As}$ barrier layer used in our calculations.

\begin{tabular}{lccccc}
\hline & Electron & & \multicolumn{2}{c}{ Heavy hole } \\
$m_{w}^{*}$ & $m_{b}^{*}$ & $V_{e}(\mathrm{meV})$ & $m_{w}^{*}$ & $m_{b}^{*}$ & $V_{h}(\mathrm{meV})$ \\
\hline 0.067 & 0.093 & 313.20 & 0.51 & 0.60 & 205.20 \\
\hline
\end{tabular}

$m_{\mathrm{w}}$ and $m_{\mathrm{b}}$ are the effective masses, respectively, in the quantum well material (GaAs) and in the barrier material (AlGaAs) given in free electron mass unit $\left(m_{\mathrm{e}}\right)$ and the offset of the conduction (valence) band $V_{e(h)}$. The energy band gap of GaAs at low temperature is $E_{g}(\mathrm{GaAs})=1519 \mathrm{meV}$.

$$
\begin{aligned}
& \Delta_{X^{+}}=E_{X^{+}}-E_{X}=J_{h h}^{s}-\left|J_{e h}^{s}\right|, \\
& \Delta_{X^{-}}=E_{X^{-}}-E_{X}=J_{e e}^{s}-\left|J_{e h}^{s}\right|, \\
& \Delta_{X X}=E_{X X}-E_{X}=J_{e e}^{s}+J_{h h}^{s}-2\left|J_{e h}^{s}\right| .
\end{aligned}
$$

The new binding energies of the trions $\Delta_{c X^{+}}, \Delta_{c X^{-}}$and the biexciton $\Delta_{c X X}$, with correlation effects, are defined as follows [18]:

$$
\begin{aligned}
\Delta_{c X^{+}} & =\Delta_{X^{+}}+\delta_{c X^{+}}-\delta_{c X}, \\
\Delta_{c X^{-}} & =\Delta_{X^{-}}+\delta_{c X^{-}}-\delta_{c X}, \\
\Delta_{c X X} & =\Delta_{X X}+\delta_{c X X}-2 \delta_{c X},
\end{aligned}
$$

with $\delta_{c X}, \delta_{c X^{+}}, \delta_{c X^{-}}$, and $\delta_{c X X}$ being the correlation energies which are written in terms of $W_{i j}$ in the following form:

$$
\begin{aligned}
\delta_{c X} & =W_{e h}, \\
\delta_{c X^{+}} & =2 W_{e h}+W_{h h}, \\
\delta_{c X^{-}} & =2 W_{e h}+W_{e e}, \\
\delta_{c X X} & =\delta_{c X^{+}}+\delta_{c X^{-}},
\end{aligned}
$$

with

$$
W_{i j}=\sum_{\left(n_{i}, m_{j}\right) \neq(0,0)} \frac{\left|U_{i j}^{n_{i} m_{j}, 00}\right|^{2}}{\left(E_{0}^{i}+E_{0}^{j}\right)-\left(E_{n_{i}}^{i}+E_{m_{j}}^{j}\right)},
$$

where $U_{i j}^{n_{i} m_{j}, 00}=1 / 4 \pi \varepsilon_{o} \varepsilon_{r} \int_{\Omega_{e}} \int_{\Omega_{h}} \psi_{i}^{n_{i}}\left(\mathbf{r}_{i}\right) \psi_{i}^{0}\left(\mathbf{r}_{i}\right)\left(1 /\left|\mathbf{r}_{e}-\mathbf{r}_{h}\right|\right)$ $\psi_{j}^{m_{j}}\left(\mathbf{r}_{j}\right) \psi_{j}^{0}\left(\mathbf{r}_{j}\right) \mathrm{d} \mathbf{r}_{e} \mathrm{~d} \mathbf{r}_{h}$ and $E_{0}^{i}$ and $E_{0}^{j}$ are the energies of a single particle ( $i$ or $j$ ) of the ground state. $E_{n_{i}}^{i}$ and $E_{m_{j}}^{j}$ are the energies of the excited states defined by the indices $n_{i}$ and $m_{j}$.

\section{Modelling Results and Discussion}

3.1. Confinement Energy and Wave Function. In Figure 2, we have presented the first five calculated squared electron wave functions for a GaAs QD of $12.9 \mathrm{~nm}$ height. The plots show that the electron and hole wave functions are highly localized inside the GaAs QD. In Figure 3, we have represented the variation of electron $\left(E_{n}\right)$ and heavy hole $\left(H H_{n}\right)$ confinement energies for the states $s(n=1)$ and $p(n=2)$ as functions of the QD height $h_{\mathrm{QD}}$. From Figure 3 , the confinement energies are very sensitive to the QD height. We observe a progressive decrease of these energies when $h_{\mathrm{QD}}$ increases. This behaviour is also observed for high excited states $(n>2)$ of the GaAs QD (Table 2). The electrons' confinement energies are higher than those of heavy holes due to their lower effective mass compared to that of holes.

3.2. Excitonic Energies. It is known that exciton formation in a QD is different than in a bulk crystal. By comparing the QD size to the Bohr radius, it is possible to define three regimes: strong confinement regime, weak confinement regime, and intermediate confinement regime. In our calculations, we will employ the strong confinement approximation, where the electron-hole Coulomb interaction is considered as a small perturbation against the single-particle terms in the 
side

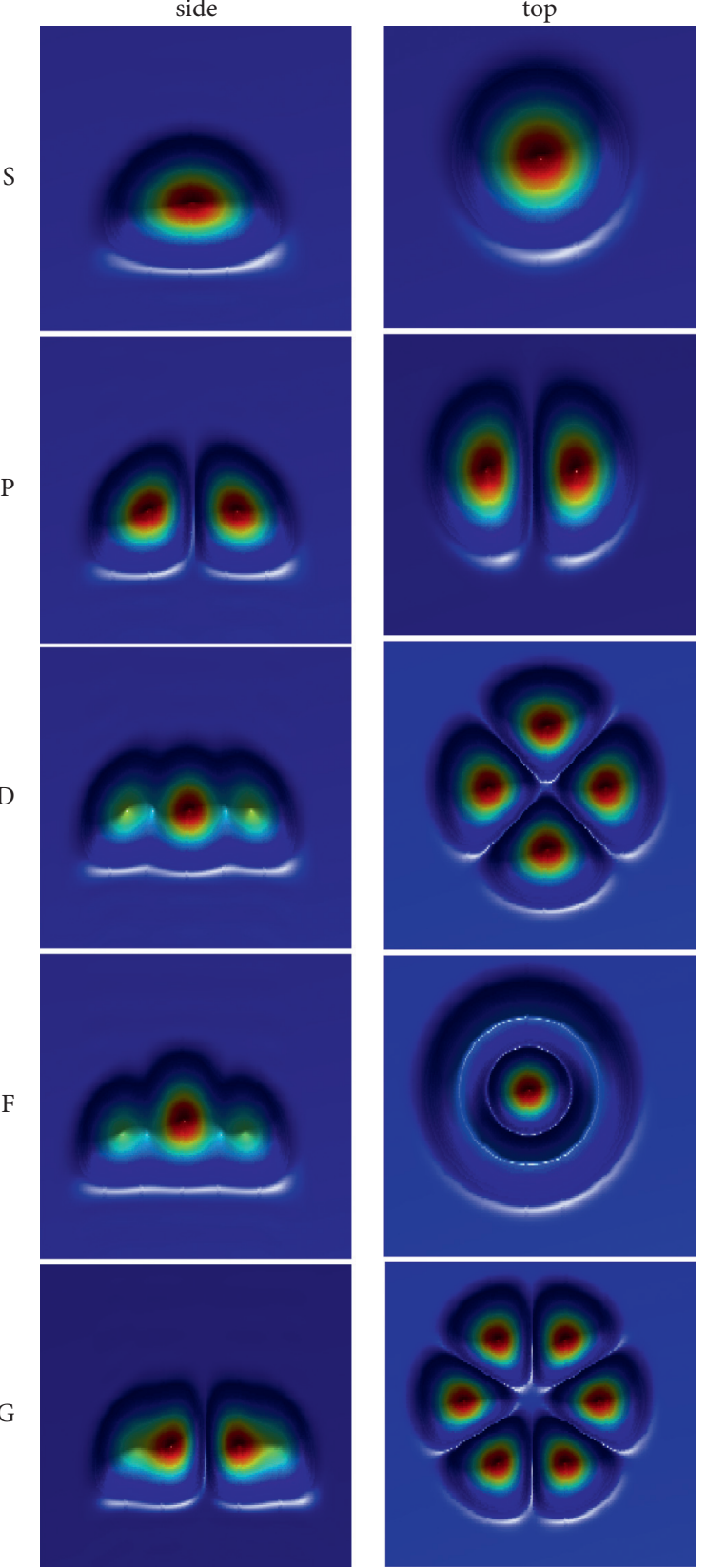

FIgURE 2: Top and side views of the probability densities of singleelectron states in a GaAs QD with $12.9 \mathrm{~nm}$ height.

Hamiltonian [9]. In our case, the nanoholes have a lateral shape with a Gaussian profile, their depth is typically $16 \mathrm{~nm}$, and their radius is around $30 \mathrm{~nm}$. The GaAs QDs have a lateral size similar to that of the nanoholes, but their height can vary between 3 and $12 \mathrm{~nm}$. Therefore, in view of the $15 \mathrm{~nm}$ exciton Bohr radius in GaAs, the confinement along the growth axis ( $z$-axis) is stronger than the lateral confinement in the $(x y)$ plane. Yet, the wave function spatial dependence in the growth direction and lateral direction is only slight, but it is significantly affected by the Coulomb interaction. Thus, $J_{e h}$ can be considered as a disturbance $[9,19]$.

In Table 3, the calculated values of $\left|J_{e h}^{s(p)}\right|$ are given as a function of QD height $h_{\mathrm{QD}}$. We note that, for $h_{\mathrm{QD}} \geq 6.4 \mathrm{~nm}$, $\left|J_{e h}^{s(p)}\right|$ increases as the height of the QDs decreases. This behaviour is similar to that observed by Abbarchi et al. [20]. The impact of the direct Coulomb interaction is more pronounced for state $s$ than for state $p$ because of the small spatial extension of the wave function of state $s$ compared to that of state $p$. From Table 3, we attribute the decrease of $\left|J_{e h}^{s(p)}\right|$, for $h_{\mathrm{QD}}<6.4 \mathrm{~nm}$, to the overlapping integrals of the wave functions associated with the electrons and holes of states $s$ and $p$. The minimum recovery is obtained for $h_{\mathrm{QD}}=6.4 \mathrm{~nm}$. Similar behaviour has been observed in selfassembled InAs/GaAs QDs [20, 21].

To investigate the accuracy of the numerical approach, the emission energies of states $s$ and $p$ are compared to those calculated by Graf et al. [1] and experimental data reported by Heyn et al. [2]. In Figure 4, we have represented the theoretical and experimental variations of the neutral exciton transition energies for the ground $\left(E_{X}^{1}\right)$ and first excited $\left(E_{X}^{2}\right)$ states as a function of $h_{\mathrm{QD}}$. In our approach, we neglected the $N$ body effects. This choice is justified based on the results of Heyn et al. [2], who showed that photoluminescence (PL) peaks shift slightly towards red by about $2 \mathrm{meV}$ when the excitation power was increased. This value remains very low compared to the confinement and Coulomb energies. Graf et al. [1] theoretically studied the optical properties of GaAs QDs. Their approach is based on the eight-band k.p model, considering $N$ body effects. Although their model is sophisticated, they neglected the band curvature of the top surface of the QD and considered it flat. This has a direct impact on the confinement energies of the carriers. However, an agreement is obtained between our theoretical results and experimental data reported by Heyn et al. as shown in Figure 4. This agreement demonstrates the suitability of our modelling approach.

3.3. Excitonic Complexes. The binding energies of the excitonic complexes in this type of QDs have been calculated. Thereby, more particular interest is given to the neutral exciton $X$, the biexciton $X X$, and the positively $X^{+}$and negatively $X^{-}$charged excitons. These excitons have been the subject of several theoretical and experimental studies for different types of self-assembled QDs such as InGaAs/GaAs $[21,22]$ and InAs/InP [23]. In these high-confinement systems, the bond energies can be determined via micro-PL spectroscopy on single dots to study the effect of size and shape on the bond energies. Unlike self-assembled InAs QDs, the addition of correlation effect, in strain-free GaAs QDs, helps to explain the binding nature of the biexciton, often observed.

Figure 5 shows the variations of the correlation energies and the bond energies of the complex excitons, with and without correlation effect, as a function of QD height $h_{\mathrm{QD}}$. The degree of correlation is specific to excitonic complexes and is sensitive to the variation of $h_{\mathrm{QD}}$. The correlation effect for a biexciton is larger due to the higher number of charge carriers involved. We also obtain $\left|\delta_{c}(X)\right|<\left|\delta_{c}\left(X^{-}\right)\right|<\left|\delta_{c}\left(X^{+}\right)\right|<\left|\delta_{c}(X X)\right|$, in agreement with the prediction established by Schliwa et al. [22]. By comparing the bond energies without and with correlation effect, we observe the formation of bound excitonic states. Indeed, 

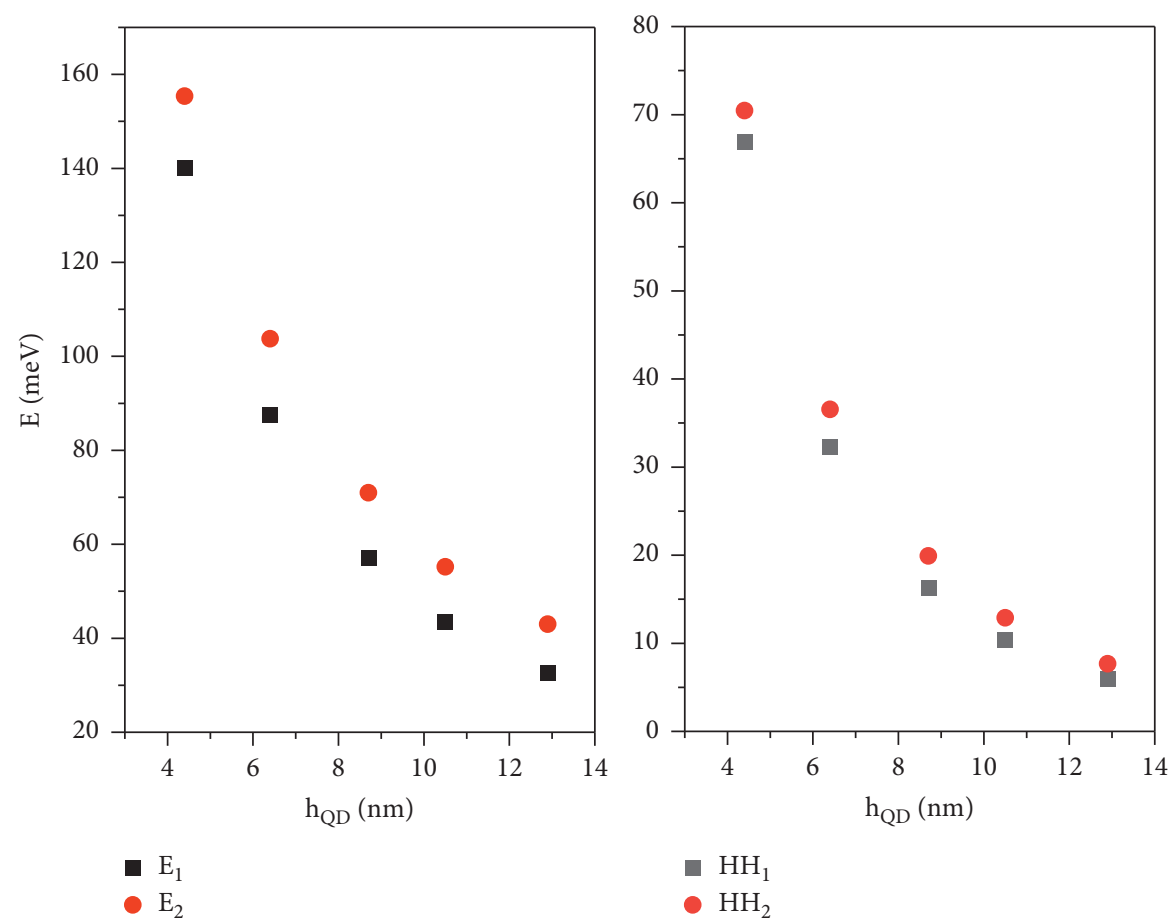

FIGURE 3: Electron $\left(E_{n}\right)$ and hole $\left(H H_{n}\right)$ confinement energies for the fundamental $s(n=1)$ and the first excited $p(n=2)$ states as a function of the QD height $h_{\mathrm{QD}}$.

TABLE 2: Energies of the ground state $E_{1}\left(H H_{1}\right)$ and the two excited states $E_{2}\left(H H_{2}\right)$ and $E_{3}\left(H H_{3}\right)$ for electrons (holes).

\begin{tabular}{lcccccc}
\hline$h_{\mathrm{QD}}(\mathrm{nm})$ & $E_{1}(\mathrm{meV})$ & $H H_{1}(\mathrm{meV})$ & $E_{2}(\mathrm{meV})$ & $H H_{2}(\mathrm{meV})$ & $E_{3}(\mathrm{meV})$ & $H H_{3}(\mathrm{meV})$ \\
\hline 4.3 & 150.8 & 71.5 & 167.0 & 77.5 & 184.4 & 119.0 \\
6.4 & 87.7 & 30.9 & 102.3 & 34.4 & 15.9 & 85.1 \\
8.6 & 56.8 & 12.9 & 70.0 & 10.2 & 67.4 & 20.1 \\
10.7 & 42.7 & 7.9 & 54.3 & 7.3 & 56.0 & 13.1 \\
12.9 & 33.6 & 5.6 & 44.0 & 9.3 \\
\hline
\end{tabular}

TABLE 3: Variation of the Coulomb interaction term $\left|J_{e h}^{s(p)}\right|$ and the overlapping integrals $\left|\left\langle S_{e} \mid S_{h}\right\rangle\right|$ and $\left|\left\langle P_{e} \mid P_{h}\right\rangle\right|$ as a function of the QD height $h_{\mathrm{QD}}$.

\begin{tabular}{lcccc}
\hline$h_{\mathrm{QD}}(\mathrm{nm})$ & $J_{e h}^{s}(\mathrm{meV})$ & $J_{e h}^{p}(\mathrm{meV})$ & $\left|\left\langle S_{e} \mid S_{h}\right\rangle\right|$ & 0.947 \\
\hline 4.4 & -14.94 & -12.26 & 0.928 & 0.896 \\
6.4 & -15.58 & -12.35 & 0.930 & 0.864 \\
8.7 & -14.34 & -11.27 & 0.956 & 0.874 \\
10.5 & -12.86 & -10.43 & 0.979 & 0.935 \\
12.9 & -11.54 & -9.78 & 0.965 \\
\hline
\end{tabular}

we underline a transition from an unbound state to a bound state for the biexciton and for the negatively charged exciton when the correlation effect is introduced. The binding energies of the biexciton obtained via our model are between -2.78 and $-5.01 \mathrm{meV}$. These values are higher in magnitude than the experimental values $(-1.3$ to $-2 \mathrm{meV})$ available in the literature $[6,24]$ for GaAs QDs based on nanoholes' shape and size. However, our results agree with those of self- assembled III-V QDs, where it is well known that the binding energy of the biexciton varies between 1 and $6 \mathrm{meV}$ $[25,26]$. The comparison between experimental and theoretical data is complex given the lack of atomic force microscopy data for the studied GaAs QDs. Our study provides a comprehensive understanding of the correlation and size effects on the final alignment of excitonic states in strain-free GaAs QDs. 


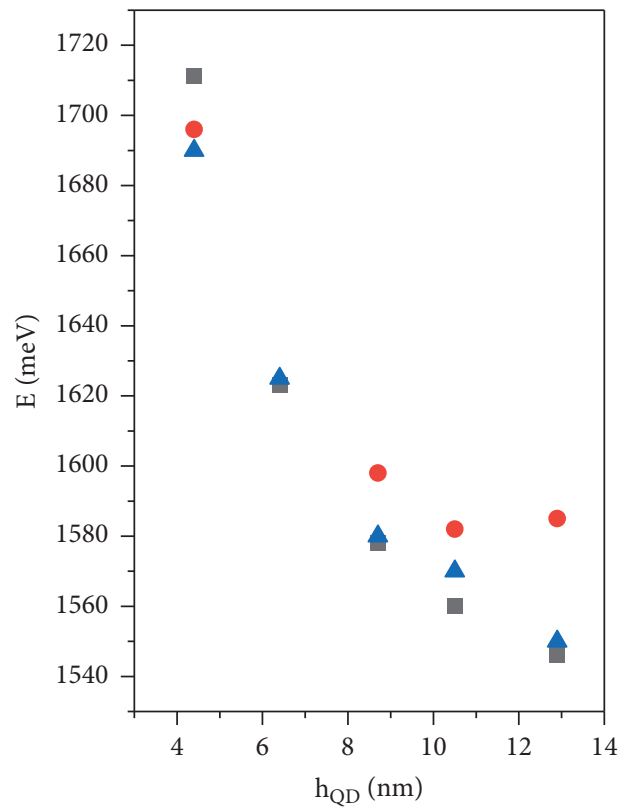

- $\mathrm{E}_{\mathrm{X}}^{1}$ our data

- $\mathrm{E}_{\mathrm{X}}^{1}$ calculated by Graf

$\Delta \mathrm{E}_{\mathrm{X}}^{1}$ measured by PL

(a)

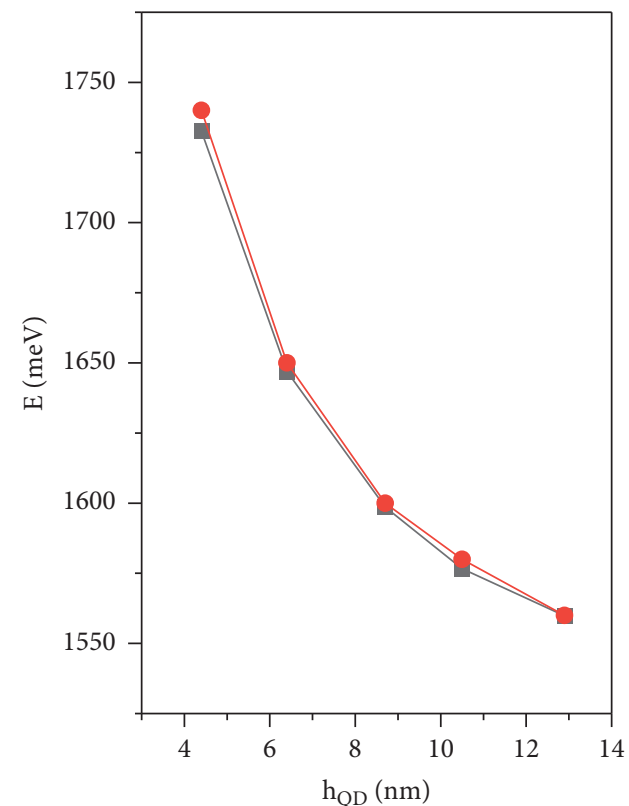

- $\mathrm{E}_{\mathrm{X}}^{2}$ our data

- $\mathrm{E}_{\mathrm{X}}^{2}$ measured by PL

Figure 4: Comparison of transition energies of the neutral exciton obtained by our model and those calculated by Graf et al. [1] and measured by Heyn et al. [2]: (a) for the ground state $E_{X}^{1}$ and (b) for the first excited state $E_{X}^{2}$.

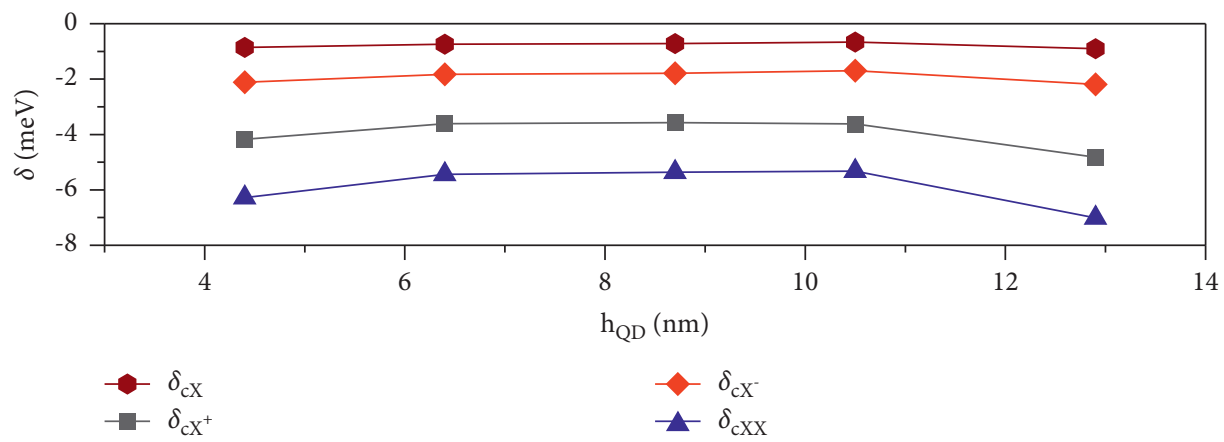

(a)

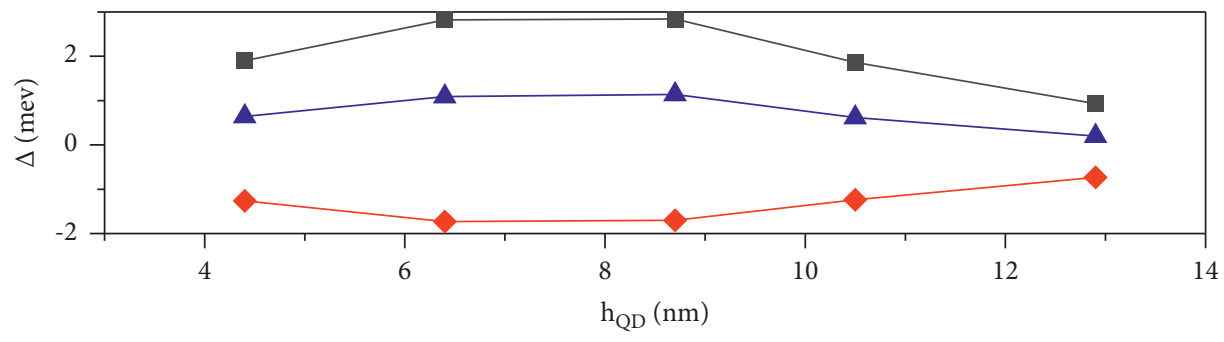

(b)

Figure 5: Continued. 


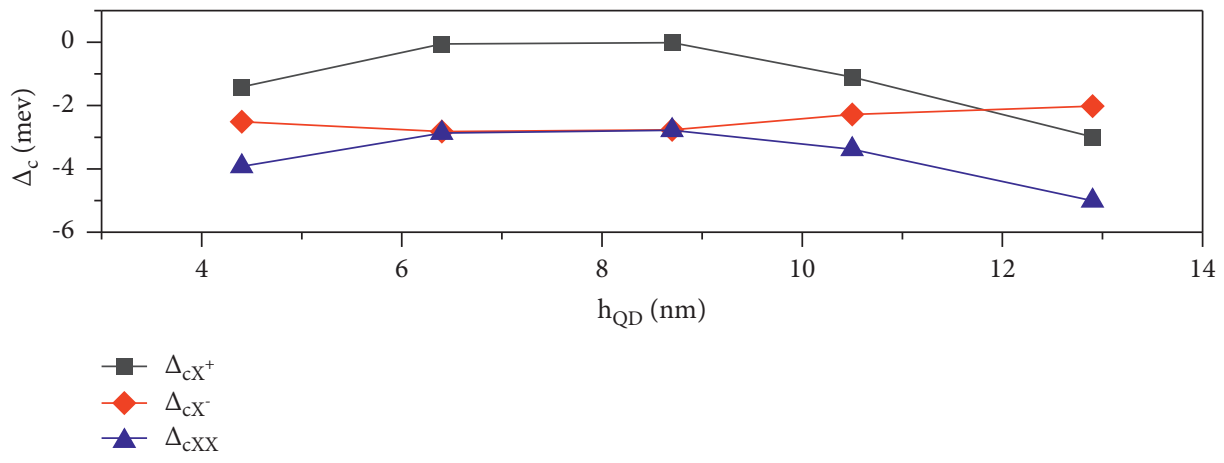

(c)

Figure 5: Variation of correlation energies $(\delta)$ of $X, X^{+}$, and $X^{-}$excitons and biexciton $X X$ as a function of $h_{\mathrm{QD}}$ (a). Bond energies of excitons $X, X^{+}$, and $X^{-}$and biexciton $X X$ as a function of $h_{\mathrm{QD}}(\mathrm{b})$ without correlation effect and (c) with correlation effect.

\section{Conclusion}

We have theoretically studied GaAs/AlGaAs QDs obtained by filling AlGaAs nanoholes with shape and profile provided by AFM measurements. The effect of size on carrier confinement, wave functions, and $s$ - $p$ splitting has been studied. The confinement energies of electrons and holes are very sensitive to the quantum dot height $h_{\mathrm{QD}}$. The excitonic energies of the $s$ and $p$ states are calculated. The impact of the direct Coulomb interaction is more pronounced for state $s$ than for $p$ due to the small spatial extension of the wave function of state $s$ compared to that of state $p$. An agreement is obtained between our theoretical results and experiment data of the literature, which indicates the suitability of our modelling approach. The behaviour of the binding energy of charged excitons $\left(X^{-}\right.$ and $X^{+}$) and biexciton $X X$ has been studied. The addition of the correlation effects allowed the formation of bound excitonic states as expected by experiments, and they are sensitive to the QD height. Our study provides a comprehensive understanding of the correlation and size effects on the final alignment of excitonic states in strainfree GaAs QDs.

\section{Data Availability}

The data used to support the findings of this study are included within the article.

\section{Conflicts of Interest}

The authors declare that they have no conflicts of interest.

\section{Acknowledgments}

This project was funded by the Deanship of Scientific Research (DSR) at King Abdulaziz University, Jeddah (Grant no. G:1715-305-1440). The authors, therefore, acknowledge with thanks the DSR for the technical and financial support.

\section{References}

[1] A. Graf, D. Sonnenberg, V. Paulava, A. Schliwa, C. Heyn, and W. Hansen, "Excitonic states in GaAs quantum dots fabricated by local droplet etching," Physical Review B, vol. 89, no. 11, Article ID 115314, 2014.

[2] C. Heyn, A. Stemmann, and W. Hansen, "Dynamics of selfassembled droplet etching," Applied Physics Letters, vol. 95, no. 17, Article ID 173110, 2009.

[3] W. Rouis, M. Haggui, S. Rekaya, L. Sfaxi et al., "Local photocurrent mapping of InAs/InGaAs/GaAs intermediate-band solar cells using scanning near-field optical microscopy," Journal of Solar Energy Materials and Solar Cells, vol. 144, pp. 324-330, 2016.

[4] A. Sayari, M. Ezzidini, B. Azeza, S. Rekaya et al., "Improvement of performance of GaAs solar cells by inserting selforganized InAs/InGaAs quantum dot superlattices," Journal of Solar Energy Materials and Solar Cells, vol. 113, pp. 1-6, 2013.

[5] A. K. Ekert, "Quantum cryptography based on bell's theorem," Physical Review Letters, vol. 67, no. 6, pp. 661-663, 1991.

[6] D. J. BenDaniel and C. B. Duke, "Space-charge effects on electron tunneling," Physical Review, vol. 152, no. 2, pp. 683-692, 1966.

[7] P. H. Lelong, O. Heller, and G. Bastard, "Multiparticle states and coulomb blockade in InAs/GaAs quantum dots," Physica E:Low-Dimensional Systems and Nanostructures, vol. 2, no. 14, pp. 678-681, 1998.

[8] J. Y. Marzin and G. Bastard, "Calculation of the energy levels in InAsGaAs quantum dots," Solid State Communications, vol. 92, no. 5, pp. 437-442, 1994.

[9] M. Grundmann, O. Stier, and D. Bimberg, "InAs/GaAs pyramidal quantum dots: strain distribution, optical phonons, and electronic structure," Physical Review B, vol. 52, no. 16, pp. 11969-11981, 1995.

[10] L. R. C. Fonseca, J. L. Jimenez, J. P. Leburton, and R. M. Martin, "Self-consistent calculation of the electronic structure and electron-electron interaction in self-assembled InAs-GaAs quantum dot structures," Physical Review B, vol. 57, no. 7, pp. 4017-4026, 1998.

[11] S. Sauvage, P. Boucaud, J. M. Gérard, and V. Thierry-Mieg, "In-plane polarized intraband absorption in InAs/GaAs 
self-assembled quantum dots," Physical Review B, vol. 58, no. 16, pp. 10562-10567, 1998.

[12] M. Baira, L. Sfaxi, L. Bouzaiene, H. Maaref, N. Chauvin, and C. Bru-Chevallier, "Broken symmetry in laterally coupled InAs/GaAs quantum dots molecule," Journal of Applied Physics, vol. 104, no. 6, Article ID 064314, 2008.

[13] A. J. Williamson and A. Zunger, "InAs quantum dots: predicted electronic structure of free-standing versus GaAsembedded structures," Physical Review B, vol. 59, no. 24, pp. 15819-15824, 1999.

[14] A. J. Williamson and A. Zunger, "Pseudopotential study of electron-hole excitations in colloidal free-standing InAs quantum dots," Physical Review B, vol. 61, no. 3, pp. 1978-1991, 2000.

[15] S. Lee, L. Jönsson, J. W. Wilkins, G. W. Bryant, and G. Klimeck, "Electron-hole correlations in semiconductor quantum dots with tight-binding wave functions," Physical Review B, vol. 63, no. 19, Article ID 195318, 2001.

[16] O. Stier, M. Grundmann, and D. Bimberg, "Electronic and optical properties of strained quantum dots modeled by 8 band k.p theory," Physical Review B, vol. 59, no. 8, pp. 5688-5701, 1999.

[17] W. J. Moore and R. T. Holm, "Infrared dielectric constant of gallium arsenide," Journal of Applied Physics, vol. 80, no. 12, pp. 6939-6942, 1996.

[18] G. A. Narvaez, G. Bester, and A. Zunger, "Excitons, biexcitons, and trions in self-assembled (In, Ga) As/GaAs quantum dots: recombination energies, polarization, and radiative lifetimes versus dot height," Physical Review B, vol. 72, no. 24, Article ID 245318, 2005.

[19] R. Seguin, A. Schliwa, S. Rodt, K. Pötschke, U. W. Pohl, and D. Bimberg, "Size-dependent fine-structure splitting in selforganized InAs/GaAs quantum dots," Physical Review Letters, vol. 95, no. 25, Article ID 257402, 2005.

[20] M. Abbarchi, T. Kuroda, T. Mano et al., "Energy renormalization of exciton complexes in GaAs quantum dots," Physical Review B, vol. 82, no. 20, Article ID 201301R, 2010.

[21] R. Neffati, I. Saïdi, S. B. Radhia, K. Boujdaria, and C. Testelin, "Effect of AlGaAs composition on the type-I to type-II transition in AlInAs/AlGaAs self-assembled quantum dots," Semiconductor Science and Technology, vol. 30, no. 8, Article ID 085008, 2015.

[22] A. Schliwa, M. Winkelnkemper, and D. Bimberg, "Fewparticle energies versus geometry and composition of $\mathrm{In}_{x} \mathrm{Ga}_{1-x} \mathrm{As} / \mathrm{GaAs}$ self-organized quantum dots," Physical Review B, vol. 79, no. 7, Article ID 075443, 2009.

[23] M. Gong, W. Zhang, G. Can Guo, and L. He, "Atomistic pseudopotential theory of optical properties of exciton complexes in InAs/InP quantum dots," Applied Physics Letters, vol. 99, no. 23, 231106 pages, 2011.

[24] C. Heyn, M. Klingbeil, C. Strelow, A. Stemmann, S. Mendach, and W. Hansen, "Single-dot spectroscopy of GaAs quantum dots fabricated by filling of self-assembled nanoholes," Nanoscale Research Letters, vol. 5, no. 10, pp. 1633-1636, 2010.

[25] K. Brunner, G. Abstreiter, G. Böhm, G. Tränkle, and G. Weimann, "Sharp-line photoluminescence and two-photon absorption of zero-dimensional biexcitons in a GaAs/ AlGaAs structure," Physical Review Letters, vol. 73, no. 8, pp. 1138-1141, 1994.

[26] A. Kuther, M. Bayer, A. Forchel, A. Gorbunov et al., "Zeeman splitting of excitons and biexcitons in single $\mathrm{In}_{0.60} \mathrm{Ga}_{0.40} \mathrm{As} /$ GaAs self-assembled quantum dots," Physical Review B, vol. 58, no. 12, pp. R7508-R7511, 1998. 